\title{
A NOTE ON GENERALIZED INDEXED NORLUND SUMMABILITY FACTOR OF AN INFINITE SERIES
}

\author{
B. P. PADHY*, P. TRIPATHY AND B. B. MISHRA \\ Department of Mathematics, School of Applied Sciences, KIIT, Deemed to be University, Bhubaneswar-24, \\ Odisha, India \\ *Corresponding author: birupakhya.padhyfma@kiit.ac.in

\begin{abstract}
In the present article, we have established a result on generalized indexed absolute Norlund summability factor by generalizing results of Mishra and Srivastava on indexed absolute Cesaro summabilty factors and Padhy et.al. on the absolute indexed Norlund summability.
\end{abstract}

\section{Introduction}

In 1930, J.M.Whittaker [18] was the 1st to establish a result on the absolute summability of Fourier series and in 1932, M. Fekete [6] established a result on generalized indexed summability. Later on the researchers like Daniel [4] in 1964, Das [5] in 1966, Siya Ram [15] in 1969, Mazhar [11] in 1971, Mishra and Srivastava [13] in 1984, Sulaiman [16] in 2011 etc. have established results on indexed summability factors of an infinite series.

Let $\sum a_{n}$ be a given infinite series with sequence of partial sums $\left\{s_{n}\right\}$. Let $t_{n}{ }^{\alpha}$ be the nth $(C, \alpha)$ mean (with order $\alpha>-1$ ) of the sequence $\left\{s_{n}\right\}$ and is given by

$$
t_{n}^{\alpha}=\frac{1}{A_{n}^{\alpha}} \sum_{k=0}^{n} A_{n-k}^{\alpha-1} s_{k}, n \in N, \text { where } A_{n}^{\alpha}=\frac{\Gamma(n+\alpha+1)}{\Gamma(\alpha+1) \Gamma(n+1)},
$$

Received 2018-11-21; accepted 2018-12-18; published 2019-03-01.

2010 Mathematics Subject Classification. 40D15, 40F05, 40G99.

Key words and phrases. absolute summability; summability factors; infinte series.

(C)2019 Authors retain the copyrights of their papers, and all open access articles are distributed under the terms of the Creative Commons Attribution License. 
then the series $\sum a_{n}$ is said to be summable $|C, \alpha|_{k}, k \geq 1$, [7] if

$$
\sum_{n=1}^{\infty}(n)^{k-1}\left|t_{n}^{\alpha}-t_{n-1}^{\alpha}\right|^{k}<\infty
$$

Let $t_{n}$ be the nth $(C, 1)$ - mean of the sequence $\left\{s_{n}\right\}$ and is given by

$$
t_{n}=\frac{1}{n+1} \sum_{k=0}^{n} s_{k}
$$

then the series $\sum a_{n}$ is said to be summable $|C, 1|_{k}, k \geq 1$, [3] if

$$
\sum_{n=1}^{\infty}(n)^{k-1}\left|t_{n}-t_{n-1}\right|^{k}<\infty
$$

Suppose $\left\{q_{n}\right\}$ be a sequence of real numbers with $q_{n}>0$, such that

$$
Q_{n}=\sum_{\nu=0}^{n} q_{\nu} \rightarrow \infty, \text { as } n \rightarrow \infty\left(Q_{-i}=q_{-i}=0, i \geq 1\right)
$$

The sequence to sequence transformation

$$
T_{n}=\frac{1}{Q_{n}} \sum_{\nu=0}^{n} q_{n-\nu} s_{\nu}
$$

defines the sequence $\left\{T_{n}\right\}$ of the $\left(N, q_{n}\right)$ - means of the sequence $\left\{s_{n}\right\}$ generated by the sequence of coefficients $\left\{q_{n}\right\}$.

The series $\sum a_{n}$ is said to be summable $\left|N, q_{n}\right|$ if the sequence $\left\{T_{n}\right\}$ is of bonded variation i.e; $\sum\left|T_{n}-T_{n-1}\right|$ is convergent.

The series $\sum a_{n}$ is said to be summable $\left|N, q_{n}\right|_{k}, k \geq 1$,if (see [8])

$$
\sum_{n=1}^{\infty}\left(\frac{Q_{n}}{q_{n}}\right)^{k-1}\left|T_{n}-T_{n-1}\right|^{k}<\infty
$$

Clearly, $\left|N, q_{n}\right|_{k}$-summabiity is same as $|C, 1|$-summabiity,when $q_{n}=1$, for all values of n. Further any sequence $\left\{\alpha_{n}\right\}$ of positive numbers the series $\sum a_{n}$ is said to be summable $\left|N, q_{n}, \alpha_{n}\right|_{k}, k \geq 1$ if

$$
\sum_{n=1}^{\infty}\left(\alpha_{n}\right)^{k-1}\left|T_{n}-T_{n-1}\right|^{k}<\infty
$$

and is said to be summable $\left|N, q_{n}, \alpha_{n} ; \delta\right|_{k}, k \geq 1, \delta \geq 0$ if

$$
\sum_{n=1}^{\infty}\left(\alpha_{n}\right)^{\delta k+k-1}\left|T_{n}-T_{n-1}\right|^{k}<\infty
$$

For any sequence $\left\{\mu_{n}\right\}, \sum_{n=1}^{\infty} a_{n} \mu_{n}$ is an infinite series.

We define

$$
\Delta \mu_{n}=\mu_{n}-\mu_{n-1},\left|\Delta \mu_{n}\right|=\left|\mu_{n}-\mu_{n-1}\right|
$$

Also, for any sequence $\left\{\mu_{n}\right\}$, by $\mu_{n}=O(n)$, we mean that the sequence $\left\{\frac{\mu_{n}}{n}\right\}$ is bounded. 


\section{KNOWN THEOREMS}

Concerning with $|C, 1|$ and $\left|N, q_{n}\right|$ summability Kishore [10] has proved the following theorem:

Theorem 2.1. Let $q_{0}>0, q_{n} \geq 0$ and $\left(q_{n}\right)$ be a non-decreasing sequence. If $\sum a_{n}$ is summable $|C, 1|$ then the series $\sum a_{n} Q_{n}(n+1)^{-1}$ is summable $\left|N, q_{n}\right|$.

Later on Ram [15] has proved the following theorem related to absolute Norlund factors of infinite series.

Theorem 2.2. Let $\left(q_{n}\right)$ be a non-increasing sequence with $q_{0}>0, q_{n} \geq 0$. If

$$
\sum_{k=1}^{n} \frac{1}{k}\left|s_{k}\right|=O\left(Y_{n}\right) \text { as } n \rightarrow \infty ;
$$

where $\left(Y_{n}\right)$ is a positive non-decreasing sequence and $\left(\mu_{n}\right)$ is a sequence such that

$$
\begin{aligned}
& \sum_{n=1}^{\infty} n\left|\Delta^{2} \mu_{n}\right| Y_{n}<\infty ; \\
& \left|\mu_{n}\right| Y_{n}=O(1) \text { as } n \rightarrow \infty,
\end{aligned}
$$

then the series $\sum a_{n} Q_{n}(n+1)^{-1}$ is summable $\left|N, q_{n}\right|$.

Also verma [17] has proved the following summability factor theorem:

Theorem 2.3. Let $\left(q_{n}\right)$ be a non-increasing sequence with $q_{0}>0, q_{n} \geq 0$. If $\sum a_{n}$ is summable $|C, 1|_{k}$ then the series $\sum a_{n} Q_{n}(n+1)^{-1}$ is summable $\left|N, q_{n}\right|_{k}, k \geq 1$.

In 1984, Mishra and Srivatava [13] proved the following theorem for $|C, 1|_{k}$ summability.

Theorem 2.4. Let $\left(Y_{n}\right)$ be a positive non-decreasing sequence and let there be sequnces $\left\{\beta_{n}\right\}$ and $\left\{\mu_{n}\right\}$ such that

$$
\begin{aligned}
& \left|\Delta \mu_{n}\right| \leq \beta_{n} ; \\
& \beta_{n} \rightarrow 0 \text { as } n \rightarrow \infty ; \\
& \left|\mu_{n}\right| Y_{n}=O(1) \text { as } n \rightarrow \infty ; \\
& \sum_{n=1}^{\infty} n\left|\Delta \beta_{n}\right| Y_{n}<\infty ; \\
& \sum_{n=1}^{\infty} \frac{1}{n}\left|s_{n}\right|^{k}=O\left(Y_{m}\right) \text { as } m \rightarrow \infty,
\end{aligned}
$$

then the series $\sum_{n=1}^{\infty} a_{n} \mu_{n}$ is summable $|C, 1|_{k}, k \geq 1$. 
Very recently, Padhy et al. [14] have proved a theorem on $\left|N, q_{n}\right|_{k}$-summability by extending theorem 2.4, in the following form:

Theorem 2.5. Let for a positive non-decreasing sequence $\left(Y_{n}\right)$, there be sequences $\left\{\beta_{n}\right\}$ and $\left\{\mu_{n}\right\}$ satisfying the conditions 2.1 to 2.5 and $\left\{q_{n}\right\}$ be a sequence with $\left\{q_{n}\right\} \in R^{+}$such that

$$
\begin{aligned}
& Q_{n}=O\left(n q_{n}\right) ; \\
& \sum_{n=1}^{\infty} \frac{q_{n}}{Q_{n}}\left|s_{n}\right|^{k}=O\left(Y_{m}\right) \text { as } m \rightarrow \infty ; \\
& \frac{Q_{n-r-1}}{Q_{n}}=O\left(\frac{q_{n-r-1}}{Q_{n}} \frac{Q_{r}}{q_{r}}\right) ; \\
& \sum_{n=r+1}^{m+1}\left(\frac{Q_{n}}{q_{n}}\right)^{k-1} \frac{q_{n-r}}{Q_{n}}=O\left(\frac{q_{r}}{Q_{r}}\right),
\end{aligned}
$$

then the series $\sum_{n=1}^{\infty} a_{n} \mu_{n}$ is summable $\left|N, q_{n}\right|_{k}, k \geq 1$, where $0 \leq r \leq n$.

It should be noted that if we take $q_{n}=1 \forall n$ then condition 2.7 will be reduced to 2.5 .

In what follows, we have generalized known theorems 2.4 and 2.5 to $\left|N, q_{n}, \alpha_{n} ; \delta\right|_{k}$ - summability in the form of the following theorem after studying [1] and [2] :

\section{Main TheOrem}

Theorem 3.1. Let $\left(Y_{n}\right)$ be a positive non-decreasing sequence and there be sequences $\left\{\beta_{n}\right\}$ and $\left\{\mu_{n}\right\}$ such that the conditions 2.1 to 2.5 are satisfied.Further let $\left\{q_{n}\right\}$ be a sequence of real numbers with $q_{n}>0$, such that

$$
\begin{aligned}
& Q_{n}=O\left(n q_{n}\right) ; \\
& \sum_{n=1}^{\infty} \frac{q_{n}}{Q_{n}}\left|s_{n}\right|^{k}=O\left(Y_{m}\right) \text { as } m \rightarrow \infty ; \\
& \frac{Q_{n-r-1}}{Q_{n}}=O\left(\frac{q_{n-r-1}}{Q_{n}} \frac{Q_{r}}{q_{r}}\right) ; \\
& \sum_{n=r+1}^{m+1}\left(\alpha_{n}\right)^{\delta k+k-1} \frac{q_{n-r}}{Q_{n}}=O\left(\frac{q_{r}}{Q_{r}}\right),
\end{aligned}
$$

then the series $\sum_{n=1}^{\infty} a_{n} \mu_{n}$ is summable $\left|N, q_{n}, \alpha_{n} ; \delta\right|_{k}, k \geq 1, \delta \geq 0$.

We require the below mentioned lemma to prove our main theorem: 


\section{Lemma [5]}

Let $\left(Y_{n}\right)$ be a positive non decreasing sequence and there be sequences $\left\{\beta_{n}\right\}$ and $\left\{\mu_{n}\right\}$ such that the conditions 2.1 to 2.5 are satisfied.Then

$$
\begin{array}{r}
\beta_{n} Y_{n}=O(1) \text { as } n \rightarrow \infty \\
\sum_{n=1}^{\infty} \beta_{n} Y_{n}<\infty
\end{array}
$$

\section{Proof of the Main theorem}

Suppose $\left(\tau_{n}\right)$ refers to the $\left(N, q_{n}\right)$ - mean of the series $\sum_{n=1}^{\infty} a_{n} \mu_{n}$. Then by definition, we have

$$
\begin{gathered}
\tau_{n}=\frac{1}{Q_{n}} \sum_{r=0}^{n} q_{n-r} \sum_{s=0}^{r} a_{s} \mu_{s} \\
=\frac{1}{Q_{n}} \sum_{s=0}^{n} a_{s} \mu_{s} \sum_{r=s}^{n} q_{n-r} \\
=\frac{1}{Q_{n}} \sum_{s=0}^{n} a_{s} \mu_{s} Q_{n-s} \\
=\frac{1}{Q_{n}} \sum_{r=0}^{n} a_{r} \mu_{r} Q_{n-r}
\end{gathered}
$$

Thus

$$
\begin{aligned}
& \tau_{n}-\tau_{n-1}=\frac{1}{Q_{n}} \sum_{r=1}^{n} Q_{n-r} a_{r} \mu_{r}-\frac{1}{Q_{n-1}} \sum_{r=1}^{n-1} Q_{n-r-1} a_{r} \mu_{r} \\
& =\sum_{r=1}^{n}\left(\frac{Q_{n-r}}{Q_{n}}-\frac{Q_{n-r-1}}{Q_{n-1}}\right) a_{r} \mu_{r} \\
& =\frac{1}{Q_{n} Q_{n-1}} \sum_{r=1}^{n}\left(Q_{n-r} Q_{n-1}-Q_{n-r-1} Q_{n}\right) a_{r} \mu_{r} \\
& =\frac{1}{Q_{n} Q_{n-1}}\left[\sum_{r=1}^{n-1} \Delta\left\{\left(Q_{n-r} Q_{n-1}-Q_{n-r-1} Q_{n}\right) \mu_{r}\right\}\right] \sum_{\nu=1}^{n} a_{\nu}, \text { with } p_{0}=0 \\
& =\frac{1}{Q_{n} Q_{n-1}}\left[\sum_{r=1}^{n-1}\left(q_{n-r} Q_{n-1}-q_{n-r-1} Q_{n}\right) \mu_{r} s_{r}+\sum_{r=1}^{n-1}\left(Q_{n-r-1} Q_{n-1}-Q_{n-r-2} Q_{n}\right) \Delta \mu_{r} s_{r}\right]
\end{aligned}
$$

(By Abel's transformation)

$$
=T_{n, 1}+T_{n, 2}+T_{n, 3}+T_{n, 4}(\text { say })
$$

Now, to show $\sum_{n=1}^{\infty} a_{n} \mu_{n}$ is summable $\left|N, q_{n}, \alpha_{n} ; \delta\right|_{k}, k \geq 1, \delta \geq 0$, by 1.6, we need to show that

$$
\sum_{n=1}^{\infty}\left(\alpha_{n}\right)^{\delta k+k-1}\left|\tau_{n}-\tau_{n-1}\right|^{k}<\infty
$$


i.e; to show that

$$
\sum_{n=1}^{\infty}\left(\alpha_{n}\right)^{\delta k+k-1}\left|T_{n, 1}+T_{n, 2}+T_{n, 3}+T_{n, 4}\right|^{k}<\infty .
$$

It will be enough to show that

$$
\sum_{n=1}^{\infty}\left(\alpha_{n}\right)^{\delta k+k-1}\left|T_{n, j}\right|^{k}<\infty \text { for } j=1,2,3,4 .
$$

to establish the main theorem by using the inequality given by Minkowski.

Now we have

$$
\begin{aligned}
& \sum_{n=2}^{m+1}\left(\alpha_{n}\right)^{\delta k+k-1}\left|T_{n, 1}\right|^{k} \\
& \sum_{n=2}^{m+1}\left(\alpha_{n}\right)^{\delta k+k-1}\left|\frac{1}{Q_{n} Q_{n-1}} \sum_{r=1}^{n-1} q_{n-r} Q_{n-1} \mu_{r} s_{r}\right| \\
& \left.\leq \sum_{n=2}^{m+1}\left(\alpha_{n}\right)^{\delta k+k-1} \frac{1}{Q_{n}}\left(\sum_{r=1}^{n-1} q_{n-r}\left|\mu_{r}\right|^{k}\left|s_{r}\right|^{k}\right)\left(\frac{1}{Q_{n}} \sum_{r=1}^{n-1} q_{n-r}\right)^{k-1} \text { (Using Holder's inequality }\right) \\
& =O(1) \sum_{r=1}^{m}\left|\mu_{r}\right|^{k}\left|s_{r}\right|^{k} \sum_{n=r+1}^{m+1}\left(\alpha_{n}\right)^{\delta k+k-1}\left(\frac{q_{n-r}}{Q_{n}}\right) \\
& =O(1) \sum_{r=1}^{m}\left|\mu_{r}\right|^{k}\left|s_{r}\right|^{k} \frac{q_{r}}{Q_{r}}, \text { by } 3.4 \\
& =O(1) \sum_{r=1}^{m} \frac{q_{r}}{Q_{r}}\left|s_{r}\right|^{k}\left|\mu_{r}\right|\left|\mu_{r}\right|^{k-1} \\
& =O(1) \sum_{r=1}^{m-1} \Delta\left|\mu_{r}\right| \sum_{w=1}^{r} \frac{q_{w}}{Q_{w}}\left|s_{w}\right|^{k}+O(1)\left|\mu_{m}\right| \sum_{r=1}^{m} \frac{q_{r}}{Q_{r}}\left|s_{r}\right|^{k} \\
& =O(1) \sum_{r=1}^{m-1}\left|\Delta \mu_{r}\right| Y_{r}+O(1)\left|\mu_{m}\right| Y_{m}, \text { by } 3.2 \\
& =O(1), \text { as } m \rightarrow \infty
\end{aligned}
$$

(By the lemma and 2.3)

Next,

$$
\begin{aligned}
& \sum_{n=2}^{m+1}\left(\alpha_{n}\right)^{\delta k+k-1}\left|T_{n, 2}\right|^{k} \\
& =\sum_{n=1}^{m+1}\left(\alpha_{n}\right)^{\delta k+k-1}\left|\frac{1}{Q_{n} Q_{n-1}} \sum_{r=1}^{n-1} q_{n-r-1} Q_{n} \mu_{r} s_{r}\right|^{k} \\
& \leq \sum_{n=2}^{m+1}\left(\alpha_{n}\right)^{\delta k+k-1} \frac{1}{Q_{n-1}}\left(\sum_{r=1}^{n-1} q_{n-r-1}\left|\mu_{r}\right|^{k}\left|s_{r}\right|^{k}\right)\left(\frac{1}{Q_{n-1}} \sum_{r=1}^{n-1} q_{n-r-1}\right)^{k-1}
\end{aligned}
$$




$$
\begin{aligned}
& =O(1) \sum_{r=1}^{m}\left|\mu_{r}\right|^{k}\left|s_{r}\right|^{k} \sum_{n=r+1}^{m+1}\left(\alpha_{n}\right)^{\delta k+k-1}\left(\frac{q_{n-r-1}}{Q_{n-1}}\right) \\
& =O(1) \sum_{r=1}^{m}\left|\mu_{r}\right|^{k}\left|s_{r}\right|^{k} \frac{q_{r}}{Q_{r}} \\
& =O(1), \text { as } m \rightarrow \infty, \text { As in proof of the 1st part. }
\end{aligned}
$$

Further,

$$
\begin{aligned}
& \sum_{n=2}^{m+1}\left(\alpha_{n}\right)^{\delta k+k-1}\left|T_{n, 3}\right|^{k} \\
& =\sum_{n=1}^{m+1}\left(\alpha_{n}\right)^{\delta k+k-1}\left|\frac{1}{Q_{n} Q_{n-1}} \sum_{r=1}^{n-1} Q_{n-r-1} Q_{n-1} \Delta \mu_{r} s_{r}\right| \\
& \leq \sum_{n=2}^{m+1}\left(\alpha_{n}\right)^{\delta k+k-1} \frac{1}{Q_{n}}\left(\sum_{r=1}^{n-1} Q_{n-r-1}\left|\Delta \mu_{r}\right|\left|s_{r}\right|^{k}\right)\left(\frac{1}{Q_{n}} \sum_{r=1}^{n-1} Q_{n-r-1}\left|\Delta \mu_{r}\right|\right)^{k-1} \\
& \operatorname{Since},\left(\frac{1}{Q_{n}} \sum_{r=1}^{n-1} Q_{n-r-1}\left|\Delta \mu_{r}\right|\right) \leq \sum_{r=1}^{n-1}\left|\Delta \mu_{n}\right| \leq n\left|\Delta \mu_{r}\right| \leq n \beta_{n}
\end{aligned}
$$

Therefore,

$$
\begin{aligned}
& \sum_{n=2}^{m+1}\left(\alpha_{n}\right)^{\delta k+k-1}\left|T_{n, 3}\right|^{k} \\
& \leq O(1) \sum_{r=1}^{m}\left(r \beta_{r}\right)^{k-1}\left|\Delta \mu_{r}\right|\left|s_{r}\right|^{k} \sum_{n=r+1}^{m+1}\left(\alpha_{n}\right)^{\delta k+k-1} \frac{Q_{n-r-1}}{Q_{n}} \\
& =O(1) \sum_{r=1}^{m}\left|\Delta \mu_{r}\right|\left|s_{r}\right|^{k} \frac{q_{r}}{Q_{r}} \\
& \leq O(1) \sum_{r=1}^{m} \beta_{r}\left|s_{r}\right|^{k} \frac{q_{r}}{Q_{r}} \\
& =O(1) \sum_{r=1}^{m-1} \Delta\left(\beta_{r}\right) \sum_{w=1}^{r} \frac{q_{w}}{Q_{w}}\left|s_{w}\right|^{k}+O(1)\left(\beta_{m}\right) \sum_{r=1}^{m} \frac{q_{r}}{Q_{r}}\left|s_{r}\right|^{k} \\
& =O(1) \sum_{r=1}^{m-1}\left|\Delta \beta_{r}\right| Y_{r}+O(1)\left(\beta_{m}\right) Y_{m} \\
& =O(1) \text { as } m \rightarrow \infty
\end{aligned}
$$

Now,

$$
\begin{aligned}
& \sum_{n=2}^{m+1}\left(\alpha_{n}\right)^{\delta k+k-1}\left|T_{n, 4}\right|^{k} \\
& =\sum_{n=2}^{m+1}\left(\alpha_{n}\right)^{\delta k+k-1}\left|\frac{1}{Q_{n} Q_{n-1}} \sum_{r=1}^{n-1} Q_{n-r-2} Q_{n} \Delta \mu_{r} s_{r}\right|^{k}
\end{aligned}
$$




$$
\begin{aligned}
& \leq \sum_{n=2}^{m+1}\left(\alpha_{n}\right)^{\delta k+k-1} \frac{1}{Q_{n-1}}\left(\sum_{r=1}^{n-1} Q_{n-r-2}\left|\Delta \mu_{r}\right|\left|s_{r}\right|^{k}\right) \frac{1}{Q_{n-1}} \sum_{r=1}^{n-1} Q_{n-r-2}\left|\Delta \mu_{r}\right| \\
& =O(1) \sum_{r=1}^{m}\left(r \beta_{r}\right)^{k-1}\left|\Delta \mu_{r}\right|\left|s_{r}\right|^{k} \sum_{n=r+1}^{m+1}\left(\alpha_{n}\right)^{\delta k+k-1}\left(\frac{Q_{n-r-1}}{Q_{n}}\right), \text { (as above) } \\
& =O(1) \sum_{r=1}^{m}\left|\Delta \mu_{r}\right|\left|s_{r}\right|^{k} \frac{q_{r}}{Q_{r}} \\
& =O(1) \text { as } m \rightarrow \infty . \text { (as above) }
\end{aligned}
$$

This completes the proof of the theorem.

\section{Conclusion}

If $\left(Y_{n}\right)$ is a positive non-decreasing sequence and there be sequences $\left\{\beta_{n}\right\}$ and $\left\{\mu_{n}\right\}$ such that the conditions 2.1 to 2.5 along with the conditions 4.1 and 4.2 are satisfied then the series $\sum_{n=1}^{\infty} a_{n} \mu_{n}$ is summable $\left|N, q_{n}, \alpha_{n} ; \delta\right|_{k}, k \geq 1, \delta \geq 0$, under the conditions 3.1 to 3.4. Thus, our result generalizes the result of Mishra and Srivastava [13] and Padhy et. al [14].

\section{REFERENCES}

[1] Bor, H., A note on two summability methods, Proc. Amer. Math. Soc., 98(1986), 81-84.

[2] Bor, H., A note on absolute summability factors, Int. J. Math. And Math. Sci., 17(3), (1994), 479-482.

[3] Bor.H, On absolute summability factors of infinite series, Rocky Mt. J. Math., 23(4)(1993), 1221-1230.

[4] Daniel, E.C., On absolute summability factors of infinite series, Proc. Japan Acad., 40(2)(1964), 65-69..

[5] Das, G, On absolute Norlund summability factors of infinite series, J. Lond. Math. Soc., 41(1966), 685-692.

[6] F. Fekete, M., On absolute summability of infinite series, Proc. Edinburgh Math. Soc., 3(2)(1932), 132-134.

[7] Flett, T.M., On an extension of absolute summability and some theorems of Little wood and Palay, Proc. Lond. Math. Soc., 7(1957), 113-141.

[8] Hardy, G.H., Divergent series, Oxford University press, (1949).

[9] Hsiang,,F.C., On absolute Norlund summability of a Fourier series, J. Austral. Math. Soc., 7(1967), 252-256.

[10] Kishore, N., On the absolute norlund summability factors, Riv. Mat. Univ. Parma, 6(1965), 129-134.

[11] Mazhar,S.M., On absolute summability factors of infinite series, Tohoku Math. J., 23(3)(2008), 433-451.

[12] Misra, U.K., Misra, M. and Padhy, B.P., On the local property of indexed Norlund summability of a factored Fourier series, Int. J. Res. Rev. App. Sci., 5(1)(2010), 52-58.

[13] Mishra, K.N and Srivastava, R.S.L., On absolute Cesaro summability factors of infinite series, Portugaliae Math., 42(1)(1983-84), 53-61.

[14] Padhy, B.P., Majhi, B., Samanta, P., Misra, M. and Misra, U.K., A note on the absolute indexed Norlund summability, New trends Math. Sci., 6(4), (2018), 54-59.

[15] Ram, S., On the absolute norlund summability factors of infinite series, Indian J. Pure Appl. Math., 2(1971), 275-282.

[16] Sulaiman, W.T., On some absolute summability factors of infinite series, Gen. Math. Notes, 2(2)(2011), 7-13.

[17] Verma, R.S., On the absolute Norlund summability factors, Riv. Math. Univ. Parma, 3(1977), 27-33.

[18] Whittaker, J.M., The absolute summability of Fourier series, Proc. Edinburgh Math. Soc., 2(2)(1930), 1-5. 